Fabr i cat i on of magnet omet er s wi th mul t i pl e- SQul D ar r ays

\begin{tabular}{|l|l|}
\hline 著者 & $\begin{array}{l}\text { NATSUDA M zushi, NAKAMRA K, M KAM H. , } \\
\text { KURI K Shi nya }\end{array}$ \\
\hline $\begin{array}{l}\text { j our nal or } \\
\text { publ i cat i on t i t l e }\end{array}$ & I EEE transact i ons on appl i ed super conduct i vi ty \\
\hline vol une & 15 \\
\hline number & 2 \\
\hline page $r$ ange & $817-820$ \\
\hline year & $2005-06$ \\
\hline URL & ht t p: //hdl . handl e. net /10258/208 \\
\hline
\end{tabular}




\title{
Fabrication of Magnetometers With Multiple-SQUID Arrays
}

\author{
Mizushi Matsuda, Kanto Nakamura, Haruki Mikami, and Shinya Kuriki
}

\begin{abstract}
We have designed and fabricated magnetometers with multiple-SQUID arrays to achieve higher signal to noise ratio compared to that for a single SQUID. In our directly coupled scheme, ten SQUIDs are connected in parallel with the same pickup coil composed of four parallel rectangular loops. Each SQUID having inductance of $70 \mathrm{pH}$ and junctions of $1.5 \mu \mathrm{m}$ width was fabricated from an $\mathrm{Au} / \mathrm{YBa}_{2} \mathrm{Cu}_{3} \mathrm{O}_{7-\delta}$ thin film on a $\mathrm{SrTiO}_{3}$ bicrystal substrate with $30^{\circ}$ misorientation angle. To avoid forming superconducting loops between junctions of adjoining SQUIDs, a Au wiring layer interrupts a current bias line. The obtained critical current of $20 I_{\mathrm{c}}$ and normal resistance of $R_{\mathrm{n}} / 20$ including contact resistance for the Au wiring layer are $350 \mu \mathrm{A}$ and $0.4 \Omega$, respectively. The modulation voltage of $25 \mu \mathrm{V}$ for multiple-SQUIDs is the same level as for a single SQUID. On the other hand, the modulation current of $40 \mu \mathrm{A}$ is several times larger than that for a single SQUID, but rather smaller than the expected value when all SQUIDs have equal parameters. A flux noise level of $15 \mu \Phi_{0} / \mathrm{Hz}^{1 / 2}$ at $100 \mathrm{~Hz}$, including preamplifier noise, was observed by using flux-locked loop electronics in a magnetically shielded room.
\end{abstract}

Index Terms-Bicrystal junctions, directly coupled magnetometer, high-temperature superconductors, SQUID arrays.

\section{INTRODUCTION}

$\mathbf{T}$ HERE is great interest in using high- $T_{\mathrm{c}}$ dc superconducting quantum interference devices (SQUIDs) for practical applications like geophysical prospecting, biomedical investigations [1], and nondestructive evaluation [2]. Magnetometers with field sensitivities below $50 \mathrm{fT} / \mathrm{Hz}^{1 / 2}$ at $77 \mathrm{~K}$ have been reported [3], [4]. However, in the case of biomagnetic applications such as magnetocardiograms (MCG) and magnetoencephalograms (MEG), the sensitivity of high- $T_{\mathrm{c}}$ SQUIDs is not enough for clinical diagnosis.

One approach to improve the signal to noise $(S / N)$ ratio is the use of the multiple-SQUID array. When $n$ identical noninteracting dc-SQUIDs are connected in series and biased at a constant current, the voltage across the array exhibits a voltage modulation $(\Delta V)$ which is $n$ times larger in amplitude than that of a single device. Since the voltage noise of array increases as $n^{1 / 2}$ because of its uncorrelated nature, the resultant $S / N$ ratio

Manuscript received October 5, 2004. This work was supported in part by the Japan Society for Promotion of Science under Grant-in-Aid for Scientific Research (C) 15560287.

M. Matsuda and K. Nakamura are with the Muroran Institute of Technology, Muroran, Hokkaido 050-8585, Japan (e-mail: matsuda@mmm.muroran-it. ac.jp).

H. Mikami was with the Muroran Institute of Technology, Muroran, Hokkaido 050-8585, Japan. He is now with the Japan Steel Works Ltd., Muroran, Hokkaido 051-8505, Japan (e-mail: haruki_mikaki@jsw.co.jp).

S. Kuriki is with the Research Institute for Electronic Science, Hokkaido University, Sapporo, Hokkaido 060-0812, Japan (e-mail: sk@es.hokudai.ac.jp).

Digital Object Identifier 10.1109/TASC.2005.850069 increases by $n^{1 / 2}$. Series arrays of low- $T_{\mathrm{C}}$ SQUIDs have been extensively studied as current amplifiers having large dynamic range, wide bandwidth and low noise performance [5]-[9]. The output impedance of an array with more than 100 SQUIDs rises to a level practical for direct connection to room temperature electronics. Arrays of high- $T_{\mathrm{c}}$ SQUIDs with grain boundary junctions were also investigated by Lee et al. [10]. They found that improvement of modulation voltage is small due to the wide spread of critical currents $\left(I_{\mathrm{c}}\right)$ among SQUIDs. The modulation amplitude of each SQUID at the same bias current is depressed below its maximum and thus the array amplitude is suppressed. A series array with more than two SQUIDs cannot be used in a directly coupled configuration [11], [12]. Difficulty in fabrication of multilayered structures for coupling to pickup coils is one of disadvantage of series high- $T_{\mathrm{c}}$ SQUID arrays.

On the other hand, parallel arrays of SQUIDs have not been reported yet. In a parallel array, all SQUIDs can operate at the same voltage near a maximum modulation point regardless of their $I_{\mathrm{c}}$ values. Although the voltage modulation remains the same as a single device, the voltage noise of an array decreases as $n^{-1 / 2}$. Thus, an $S / N$ ratio of a parallel array with $n$ SQUIDs should scale to $n_{1 / 2}$ as in series arrays.

In this paper, we describe a magnetometer with parallel SQUID arrays. Magnetometers with 10 SQUIDs connected in parallel with the same pickup coil were made from single layer $\mathrm{YBa}_{2} \mathrm{Cu}_{3} \mathrm{O}_{7-\delta}$ (YBCO) films and bicrystal grain boundary junctions. Noise properties for different number of SQUIDs $(n)$ are also studied.

\section{DESIGN AND FABRICATION}

The magnetometers with parallel 10-SQUID arrays are prepared from c-axis oriented YBCO films. A $150 \mathrm{~nm}$ thick film of YBCO and a $50 \mathrm{~nm}$ thick film of Au were deposited sequentially without breaking vacuum by pulsed laser deposition on a $10 \mathrm{~mm}$ $\times 10 \mathrm{~mm} \mathrm{SrTiO}_{3}$ bicrystal substrate with a misorientation angle of $30^{\circ}$. The films show a transition temperature of $89 \mathrm{~K}$ and a critical current density at $77 \mathrm{~K}$ exceeding $5 \times 10^{6} \mathrm{~A} / \mathrm{cm}^{2}$ except at the grain boundaries. They were patterned to form the magnetometer geometry illustrated in Fig. 1(a) by Ar ion beam etching and standard photolithography. The rectangular pickup loop consists of 4 parallel lines of $100 \mu \mathrm{m}$ width. We formed many slots $(45 \mu \mathrm{m} \times 5 \mu \mathrm{m})$ in the pickup loop to avoid flux entry into the superconductor by reduction of the superconducting line width. An estimation for the threshold field of flux entry is given by $B_{\mathrm{T}}=$ $\left(\pi \Phi_{0}\right) /\left(4 w^{2}\right)$ [13], where $w$ is the maximum line width of the film. Corresponding $B_{\mathrm{T}}$ of $65 \mu \mathrm{T}$ for this device is rather larger than the Earth's dc field of 30-50 $\mu \mathrm{T}$. The estimated inductance $L_{\mathrm{p}}$ and an effective area $A_{\mathrm{p}}$ for our pickup loop are $5 \mathrm{nH}$ and 10 


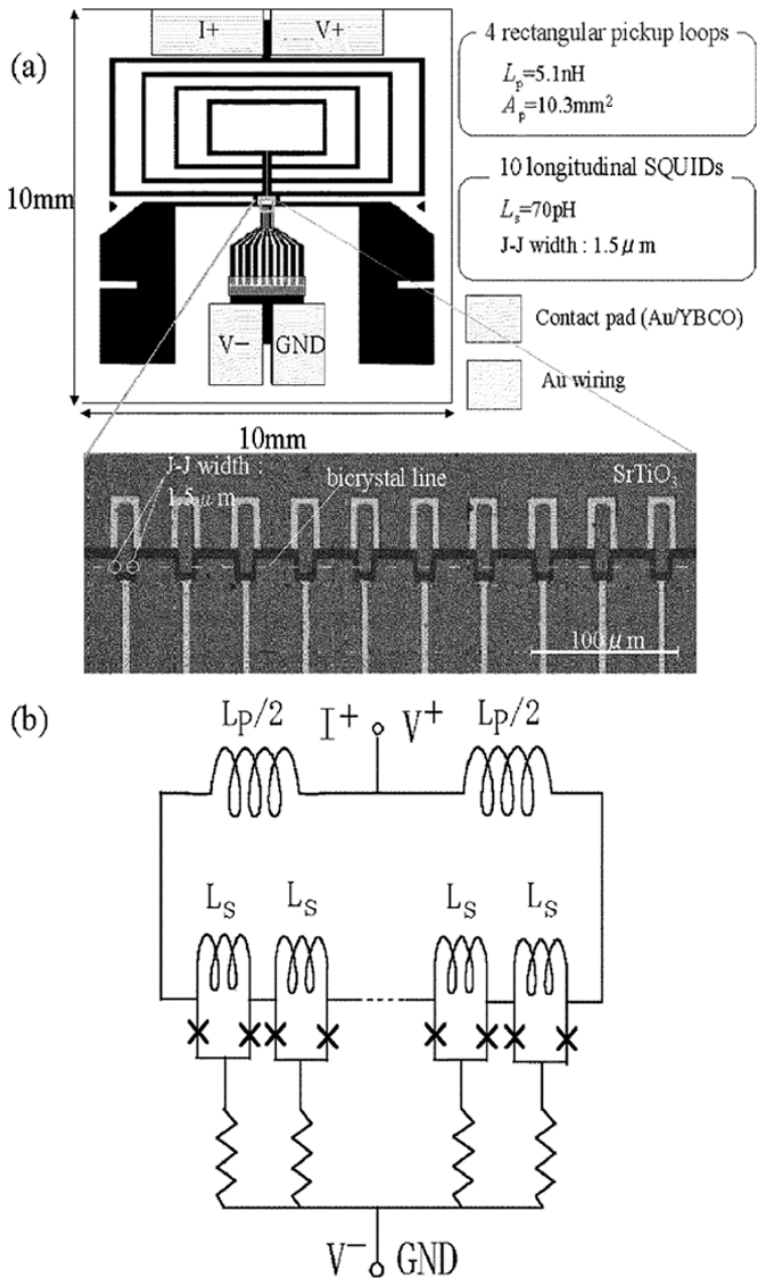

Fig. 1. (a) Layout of a magnetometer and inner part of the sensor around a parallel 10-SQUIDs array and (b) equivalent circuit of a direct coupled magnetometer with multiple-SQUIDs array.

$\mathrm{mm}^{2}$, respectively. Each SQUID of an array in the middle of the structure has two parallel strips of $5 \mu \mathrm{m}$ width and a long hole perpendicular to the bicrystal line. The hole length and width are 44 and $10 \mu \mathrm{m}$, respectively. The $1.5 \mu \mathrm{m}$ wide bridges including grain boundary junctions are located at the end of the hole. In this geometry, a high flux coupling coefficient $\alpha$ of around 0.8 was obtained [14]. The inductance value $L_{\mathrm{s}}$ of a SQUID, estimated using the coplanar stripline formula [15] that includes the kinetic inductance, is about $70 \mathrm{pH}$. An effective area of the magnetometer $A_{\text {eff }}$ given by $A_{\text {eff }}=\alpha A_{\mathrm{p}} L_{\mathrm{s}} / L_{\mathrm{p}}$ can be calculated as $0.14 \mathrm{~mm}^{2}$. To avoid interloop flux coupling, a SQUID in an array is placed far from neighboring ones by $20 \mu \mathrm{m}$. Furthermore, to avoid forming superconducting loops between junctions in adjoining SQUIDs, an additional etching process cut superconducting lines for current bias. Then, a sputter-deposited $\mathrm{Au}$ wiring layer connected them again.

\section{RESULTS AND DISCUSSION}

For the readout of a parallel array SQUID illustrated in Fig. 2(a), detection of modulation currents $(\Delta I)$ by applying field is desirable. In this configuration, we would drive all SQUIDs at the same voltage near a maximum modulation point (a)

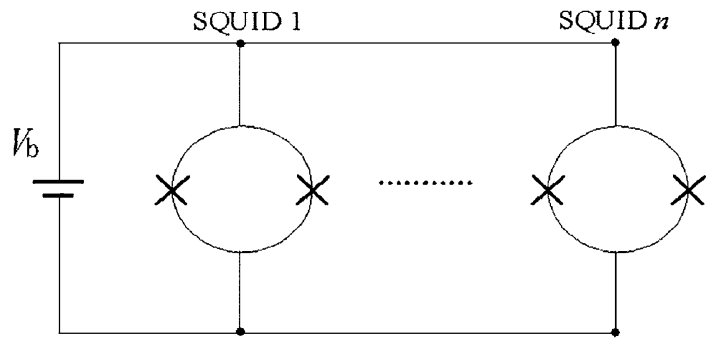

(b)

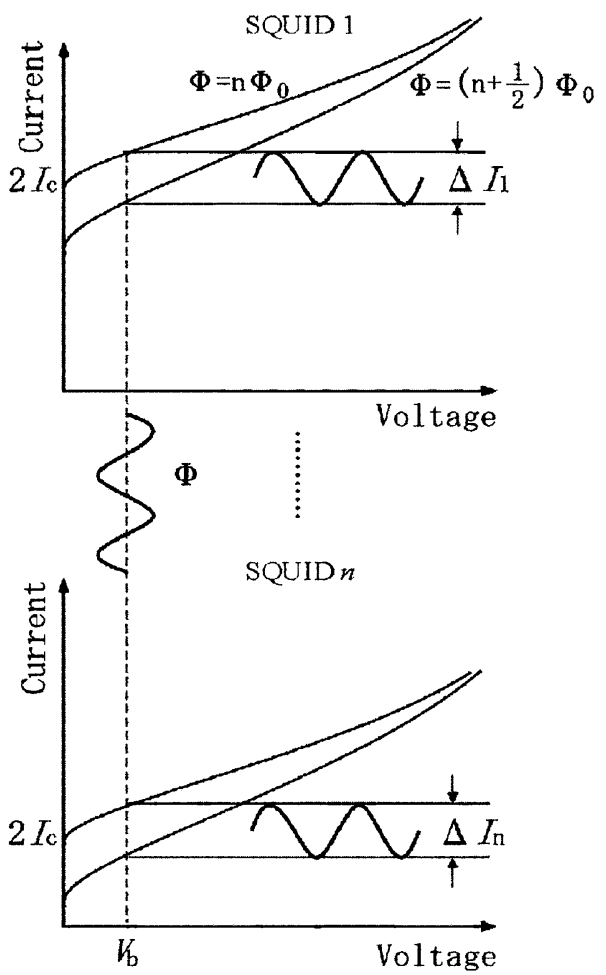

Fig. 2. (a) Schematic drawing of a parallel $n$-SQUID array and (b) principle of the detection of modulation currents for a parallel-SQUID array.

as shown in Fig. 2(b). Even if an $I_{\mathrm{c}}$ value of each SQUID is different, relatively large modulation of $\Delta I\left(=\Delta I_{1}+\cdots+\Delta I_{n}\right)$ can be obtained. It is analogous to $\Delta V$ in a series array at a constant current bias. However, in our present stage, it is rather hard to prepare a current amplifier such as the SQUID sensor itself and a current source with feedback for operation at a constant bias voltage. Thus, we measured characteristics of the magnetometers by a conventional current-bias method in this study. Fig. 1(b) shows an equivalent circuit of the magnetometer with a parallel SQUID array. The bias current injected through the pickup coil flowed from both sides of an array into the individual SQUID. All characterizations of the magnetometers including noise measurements by flux-locked loop (FLL) electronics were performed in a magnetically shielded room. Magnetic flux is applied by a short solenoid coil with $10 \mathrm{~mm}$ diameter placed just below the device. A heater resistor was also placed in order to heat the device momentarily to above its transition temperature and to remove trapped flux.

Fig. 3 shows the current-voltage $(I-V)$ characteristics for magnetometers with a parallel 10-SQUID array. The critical current of $20 I_{\mathrm{c}}$ and normal resistance of $R_{\mathrm{n}} / 20$ including resistance 


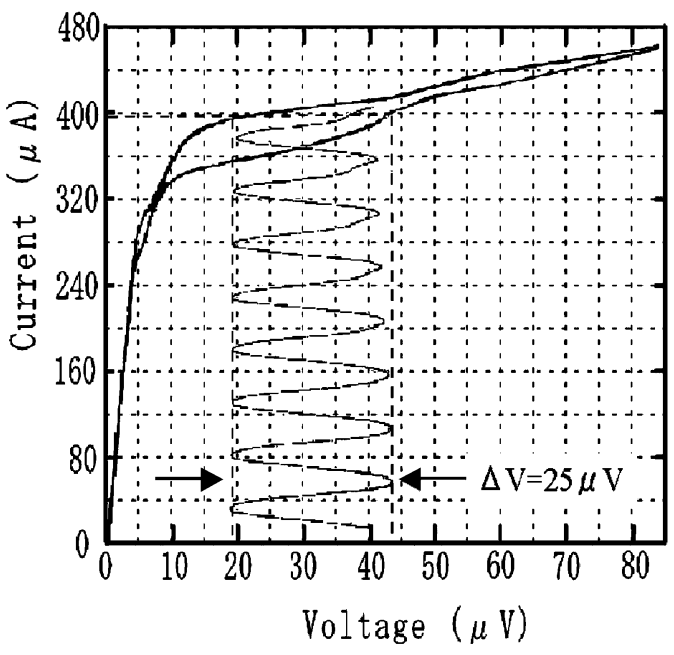

Fig. 3. $I-V$ characteristics for magnetometers with a parallel 10-SQUIDs array. A $V-\Phi$ curve obtained at a bias current around $390 \mu \mathrm{A}$ is also drawn.

for Au layer are $350 \mu \mathrm{A}$ and $0.4 \Omega$, respectively. The corresponding inductance parameter $\beta_{\mathrm{L}}=2 L_{\mathrm{s}} I_{\mathrm{c}} / \Phi_{0}$ as an average per SQUID is about 1.2. The resistance for the Au wiring layer that is evaluated by a slope in the $I-V$ curve at very low current region is $0.02 \Omega$. Most of this resistance seems to be attributed to the contact between superconducting lines and the Au layer, because the measured resistivity of $1.2 \mu \Omega \cdot \mathrm{cm}$ for our Au film at $77 \mathrm{~K}$ was too small to be responsible. A voltage-flux $(V-\Phi)$ curve obtained at a bias current around $390 \mu \mathrm{A}$ is also drawn in this figure. The maximum $\Delta V$ of $25 \mu \mathrm{V}$ is the same level as that for a single SQUID. We can see amplitude modulation of $\Delta V$ probably arising from variations in the coupling inductance $\alpha L_{\mathrm{s}}$ among 10 SQUIDs as in a series array [7], [10]. Here, $\alpha$ denotes the part of the SQUID inductance that carries the screening current coming from the pickup coil. Small difference in the $V-\Phi$ period for each SQUIDs originating from variations in $\alpha L_{\mathrm{s}}$ leads to a beat of the modulation. On the other hand, the modulation depth for currents $(\Delta I)$ of $40 \mu \mathrm{A}$ is several times larger than that for a single SQUID, but smaller than the expected value when all SQUIDs have equal parameters.

In our parallel SQUID configuration, device operation is rather complicated. Bias current flows through the coupling inductance as the screening current does and generates an additional flux into each SQUID. The resultant phase incoherence of the modulation can reduce the amplitude by canceling signals of different SQUIDs. Furthermore, re-distribution of bias current to the individual SQUID takes place dynamically by applying field. For comparison and simplification of an analysis, we also prepared 6-SQUIDs and 2-SQUIDs arrays by remaining SQUIDs from an array in sequence. Fig. 4(a), (b), and (c) show the $I-V$ and $V-\Phi$ characteristics of magnetometers for parallel 10-, 6-, and 2-SQUIDs, respectively. Modulation period remains almost the same independent of the SQUID number of $n$. Significant degradation of $\Delta V$ and $I_{\mathrm{c}} R_{\mathrm{n}}$ product values are observed for the 6-and 2-SQUID. We have not clarified the reason for such degradation, but it is likely that there is physical damage during the etching process used. It seems that only a few SQUIDs work together due to a large spread in junction parameters after etching process. The obtained parameters for magnetometers with parallel $n$-SQUIDs arrays are listed in Table I.
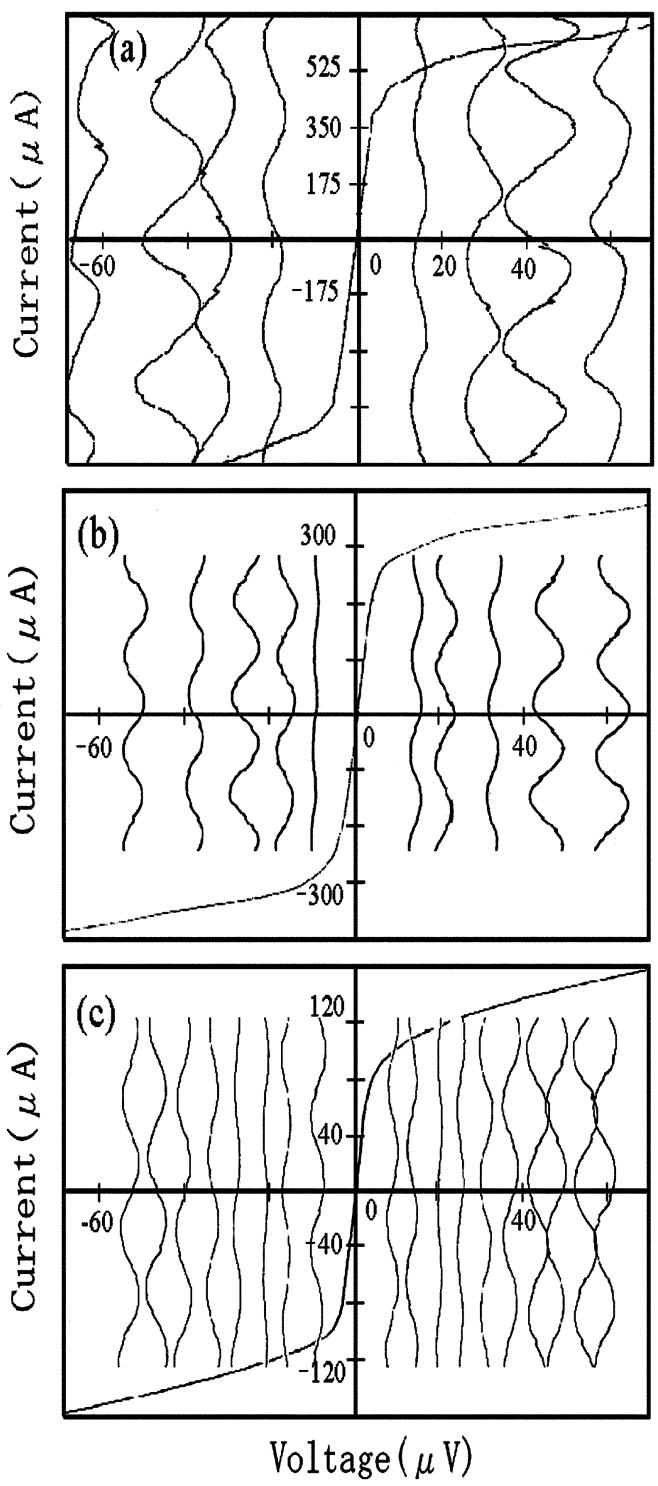

Fig. 4. I-V characteristics for magnetometers with a parallel (a) 10-, (b) 6-, and (c) 2-SQUIDs array. $V-\Phi$ characteristics at various bias currents are also shown.

TABLE I

PERFORMANCE OF MAGNETOMETERS With MUlTiPLE-SQUIDS ARRAY

\begin{tabular}{ccccc}
\hline \hline \multicolumn{1}{c}{$n$} & $\begin{array}{c}I_{\mathrm{c}} R_{\mathrm{n}} \text { product } \\
(\mu \mathrm{A})\end{array}$ & $\begin{array}{c}\Delta V \\
(\mu \mathrm{V})\end{array}$ & $\begin{array}{c}\Phi_{\mathrm{n}} \text { at } 100 \mathrm{~Hz} \\
\left(\Phi_{0} / \mathrm{Hz}^{-1 / 2}\right)\end{array}$ & $\begin{array}{c}V_{\mathrm{n}} \text { at } 100 \mathrm{~Hz} \\
\left(\mathrm{~V} / \mathrm{Hz}^{-1 / 2}\right)\end{array}$ \\
\hline 10-SQUIDs & 180 & 25 & $1.5 \times 10^{-5}$ & $1.2 \times 10^{-9}$ \\
6-SQUIDs & 140 & 9 & $4.7 \times 10^{-5}$ & $1.3 \times 10^{-9}$ \\
2-SQUIDs & 64 & 7 & $1.1 \times 10^{-4}$ & $2.4 \times 10^{-9}$ \\
\hline \hline
\end{tabular}

Flux noise spectra of magnetometers with 10-, 6-, and 2-SQUIDs measured by FLL electronics are shown in Fig. 5(a), (b), and (c), respectively. A white noise level of $15 \mu \Phi_{0} / \mathrm{Hz}^{1 / 2}$ at $100 \mathrm{~Hz}$, including preamplifier noise, was observed for the device with a 10-SQUID array. Observed flux noise $\left(\Phi_{\mathrm{n}}\right)$ and voltage noise $\left(V_{\mathrm{n}}\right)$ levels at $100 \mathrm{~Hz}$ for different $n$ are also summarized in Table I. Lower voltage noise level was obtained for larger number of $n$. For the magnetometer with a 10-SQUIDs array, $V_{\mathrm{n}}$ value of $1.2 \mathrm{nV} / \mathrm{Hz}^{1 / 2}$ is almost the same level as that of preamplifier. 


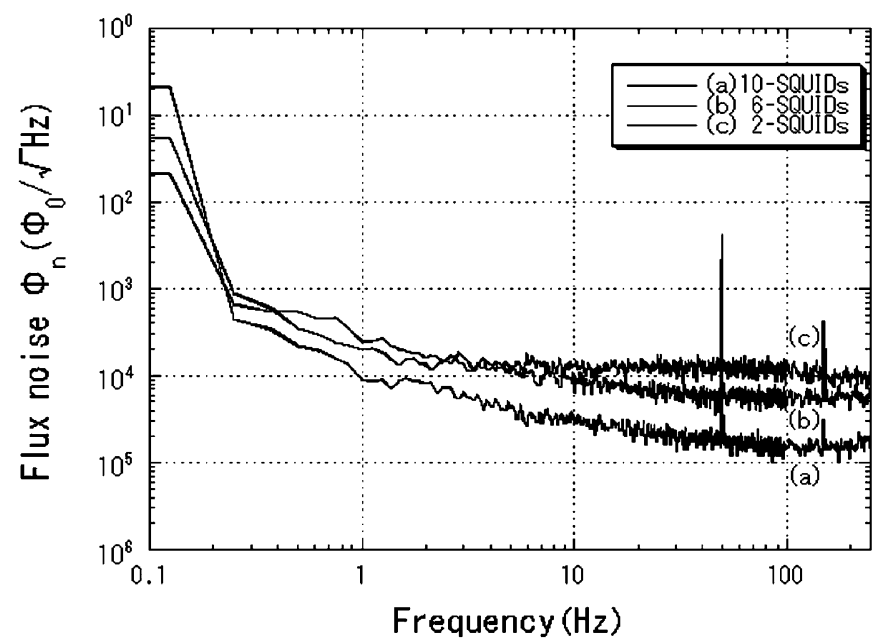

Fig. 5. Flux noise spectra of magnetometers with a (a) 10-, (b) 6-, and (c) 2-SQUIDs array measured by FLL electronics.

\section{CONCLUSION}

We have fabricated and characterized the magnetometer with a parallel multiple-SQUID array. Although the modulation voltage for multiple-SQUIDs is the same level as for a single SQUID, the modulation depth for currents is several times larger than that for a SQUID. White noise level of $\Phi_{\mathrm{n}}$ at $100 \mathrm{~Hz}$ in FLL operation and $V_{\mathrm{n}}$ calculated depends upon the SQUID number $n$ in a array, lower noise level for larger $n$.

\section{REFERENCES}

[1] K. Enpuku, D. Kuroda, T. Q. Yang, and K. Yoshinaga, "High $T_{\mathrm{c}}$ SQUID system and magnetic marker for biological immunoassays," IEEE Trans. Appl. Supercond., vol. 13, pp. 371-376, 2003.

[2] Y. Hatsukade, M. S. Aly-Hassan, N. Kasai, H. Takashima, H. Hatta, and A. Ishiyama, "SQUID-NDE method on damaged area and damage degree of defects in composite materials," IEEE Trans. Appl. Supercond., vol. 13, pp. 207-210, 2003.
[3] L. P. Lee, J. Vinetskiy, and R. Cantor, "Low-noise $\mathrm{YBa}_{2} \mathrm{Cu}_{3} \mathrm{O}_{7-\delta}$ direct-current superconducting quantum interference devices," Appl. Phys. Lett., vol. 66, pp. 1539-1541, 1995.

[4] D. Koelle, R. Kleiner, F. Ludwig, E. Dantsker, and J. Clarke, "Hightransition-temperature superconducting quantum interference devices," Rev. Mod. Phys., vol. 71, pp. 631-680, 1999.

[5] R. P. Welty and J. M. Martinis, "Two-stage integrated SQUID amplifier with series array output," IEEE Trans. Appl. Supercond., vol. 3, pp. 2605-2608, 1993.

[6] V. Foglietti, K. G. Stawiasz, M. B. Ketchen, and R. H. Koch, "Performance of a flux locked series SQUID," IEEE Trans. Appl. Supercond., vol. 13, pp. 207-210, 1993.

[7] K. Li and S. P. Hubbell, "Measurement and simulation of the voltage-flux transfer function of SQUID arrays," IEEE Trans. Appl. Supercond., vol. 5, pp. 3255-3258, 1995.

[8] F. Hirayama, N. Kasai, and M. Koyanagi, "Design of series SQUID array suppressing Josephson oscillation interference between elementSQUIDs," IEEE Trans. Appl. Supercond., vol. 9, pp. 2923-2926, 1999.

[9] M. E. Huber, P. A. Neil, R. G. Benson, D. A. Burns, A. M. Corey, C. S. Flynn, Y. Kitaygorodskaya, O. Massihzadeh, J. M. Martinis, and G. C. Hilton, "Dc SQUID series array amplifiers with $120 \mathrm{MHz}$ bandwidth (corrected)," IEEE Trans. Appl. Supercond., vol. 11, pp. 4048-4053, 2001.

[10] S.-G. Lee, Y. Huh, G.-S. Park, I.-S. Kim, Y. K. Park, and J.-C. Park, "Serial array high $T_{\mathrm{c}}$ SQUID magnetometer," IEEE Trans. Appl. Supercond., vol. 7, pp. 3347-3350, 1997.

[11] V. Schultze, V. Zakosarenko, R. IJsselsteijn, J. Ramos, and H.-G. Meyer, "Integration of multiple SQUID arrangements in single layer high $T_{c}$ magnetometers," IEEE Trans. Appl. Supercond., vol. 9, pp. 3279-3282, 1999.

[12] A. Tsukamoto, Y. Soutome, T. Fukazawa, and K. Takagi, "Dual bias current operation os 2-SQUID directly coupled magnetometers," IEEE Trans. Appl. Supercond., vol. 11, pp. 1106-1109, 2001.

[13] E. Dantsker, S. Tanaka, and J. Clarke, "High-Tc superconducting quantum interference devices with slots or holes: low $1 / f$ noise in ambient magnetic fields," Appl. Phys. Lett., vol. 70, pp. 2037-2039, 1997.

[14] M. Maruyama, S. Kuriki, Y. Yokosawa, R. S. Ahmad, D. Suzuki, and M. Koyanagi, "Flux coupling in the direct-coupled high- $T_{\mathrm{c}}$ superconducting interference devices," Jpn. J. Appl. Phys., vol. 37, pp. L722-L724, 1998.

[15] K. Yoshida, M. S. Hossain, T. Kisu, K. Enpuku, and K. Yamafuji, "Modeling of kinetic-inductance coplanar stripline with $\mathrm{NbN}$ thin films," Jpn. J. Appl. Phys., vol. 31, pp. 3844-3850, 1992. 